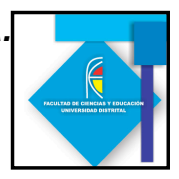

\title{
MENTEFACTO CONCEPTUAL PARA LA FUNCIÓN DE ONDA DE LA MECÁNICA CUÁNTICA
}

\section{CONCEPTUAL MIND-FACT FOR THE WAVE FUNCTION OF QUANTUM MECHANICS}

\section{Resumen}

Elisa Guevara M. ${ }^{1}$ Maureen Everth T. ${ }^{2}$ Orlando Organista $\mathrm{R}^{3}$

La mecánica cuántica representa un reto para la actual investigación en la enseñanza de la física, en gran medida, debido a que los fenómenos que ocurren a escala microscópica han reducido ideas fuertemente arraigadas a nuestra intuición, como es la idea del camino seguido por un cuerpo (trayectoria), la idea de ubicar un objeto en un lugar (localidad), o la idea de determinar con exactitud el resultado de una medición (determinismo). El investigador Lei Bao enfoca su estudio en varios tópicos que considera fundamentales para tener un buen entendimiento de la mecánica cuántica; uno de estos tópicos es la interpretación probabilística de la función de onda. En este artículo se lleva a cabo un análisis conceptual alrededor de la función de onda a través de un diagrama cognitivo denominado mentefacto conceptual; este ideograma ayuda a identificar y organizar nociones constitutivas y diferenciadoras implícitas en el concepto de función de onda, y se constituye en un aporte para la enseñanza-aprendizaje de este concepto.

Palabras clave: Enseñanza de la teoría cuántica, mentefacto conceptual, análisis conceptual, operaciones cognitivas, función de onda.

\begin{abstract}
:
Quantum mechanics represents a challenge for current research in physics education, largely because the phenomena that occur on a microscopic scale have reduce deeprooted intuitions, for example the idea of the path of a body (trajectory), the idea of locating an object in a place (place), or the idea of determining exactly the result of a measurement (determinism). The researcher Lei Bao focused his study on several topics that he considers essential to have a good understanding of quantum mechanics; one of these topics is the probabilistic interpretation of the wave function. In this article we conduct a conceptual analysis of the wave function through a
\end{abstract}

\footnotetext{
${ }^{1}$ Universidad Pedagógica Nacional, 2107liz@gmail.com

${ }^{2}$ Universidad Pedagógica Nacional, maureen288@hotmail.com

${ }^{3}$ Universidad Central. jorganistar@ucentral.edu.co
} 


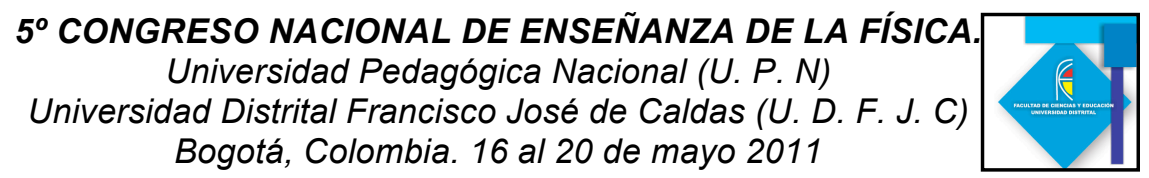

cognitive diagram called cognitive mind-fact; this ideogram helps identify and organize constituent and differentiating concepts implicit in the concept of wave function and represent a contribution to the teaching-learning concept.

Keywords: Quantum theory teaching, conceptual mind-fact, conceptual analysis, cognitive operations, wave function.

\section{INTRODUCCIÓN}

La teoría cuántica describe el comportamiento del mundo microscópico y llega a conclusiones que son contrarias a nuestra intuición (Greca, 2002). Las nuevas maneras de concebir el ámbito microscópico requiere de nuevos conceptos y principios tales como el concepto de función de onda (Bao, 1999), el de entrelazamiento (Schrödinger, 1935) y el principio de superposición de estados, entre otros.

Diversas investigaciones resaltan la importancia de la enseñanza del concepto de función de onda en mecánica cuántica y de las dificultades presentes en los estudiantes (Bao, 1999); estos estudios evidencian la necesidad de profundizar en análisis conceptuales (Organista, 2007). Miguel de Zubiría propone una metodología para analizar un concepto, la cual conduce a la elaboración de un ideograma denominado mentefacto conceptual. La importancia de realizar este tipo de ideogramas radica en que su elaboración propicia ciertas operaciones cognitivas necesarias para el aprendizaje de un concepto.

En la sección II del presente artículo se realiza una breve reseña sobre la construcción de los mentefactos conceptuales, posteriormente, en la sección III se hace una breve exposición sobre la función de onda en el marco de la teoría cuántica mostrando las proposiciones que construyen el mentefacto conceptual para la función de onda; en la sección IV se presenta el diagrama que constituye el mentefacto conceptual y finalmente, en la sección $V$, se presentan nuestras conclusiones.

\section{MENTEFACTOS: IDEOGRAMAS PARA LA REPRESENTACIÓN DEL PENSAMIENTO}

Con el objetivo de realizar acciones que contribuya al aprendizaje de conceptos, desde el constructivismo, se han desarrollado diferentes ideogramas o diagramas cognitivos que permiten sintetizar, ordenar y graficar las ideas que hay alrededor de un concepto. Miguel De Zubiría propone una representación gráfica (facto) del pensamiento que manifiesta las operaciones mentales (mente) necesarias en la organización de las proposiciones que abstraen el significado de un concepto. Este ideograma es denominado por el autor mentefacto. El pensamiento conceptual se representa mediante un ideograma llamado mentefacto conceptual y para elaborarlo es necesario conocer las proposiciones con las cuales estructurarlo, lo cual implica saber:

- ¿A qué grupo inmediatamente superior pertenece? (supraordinar).

- Qué otros conceptos son semejantes pero no son el concepto estudiado (excluir).

- Cuáles son las propiedades o cualidades del concepto (isoordinar).

- Saber la existencia de subclases del concepto (infraordinar). 


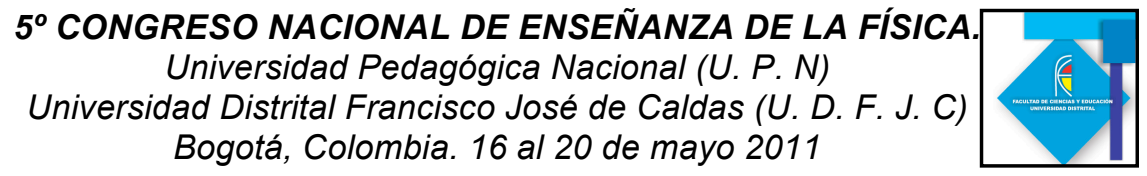

Los mentefactos conceptuales llevan a cabo dos funciones vitales, organizan las proposiciones, al hacerlo organizan nuestras mentes, y preservan el conocimiento aprendido, por lo tanto constituyen elevadas herramientas de conocimiento.

\section{LA FUNCIÓN DE ONDA EN EL MARCO DE LA TEORÍA CUÁNTICA}

La mecánica cuántica se preocupa por describir el estado de las partículas atómicas. La función ( , ) permite tener información acerca del estado de la partícula, esta función recibe el nombre de función de onda. A continuación se presentan las proposiciones con su respectivo mentefacto proposicional, para cada una de las cuatro operaciones cognitivas necesarias en la realización del mentefacto conceptual de la función de onda.

\section{a. Clase Supraordinada: Funciones de Cuadrado Integrable}

Se usarán las propiedades matemáticas de las funciones de onda para definir el conjunto al que pertenecen estas funciones, es decir, la clase supraordinada. Las funciones de onda cumplen con las siguientes propiedades matemáticas:

Proposición I: Las funciones de onda son funciones de valores en los números complejos.

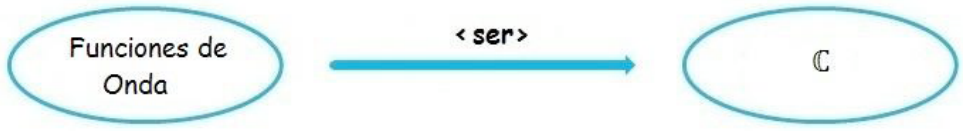

Proposición II: Las funciones de onda pertenecen al espacio 2, es decir, el cuadrado del valor absoluto de la función de onda debe ser menor que infinito. El correspondiente mentefacto sería:

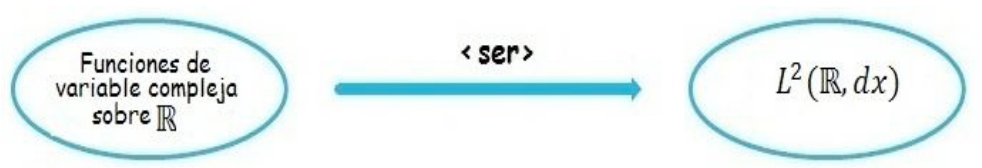

Proposición III: El Espacio 2es un de Espacio de Hilbert y sus elementos deben satisfacer la condición de integrable al cuadrado.

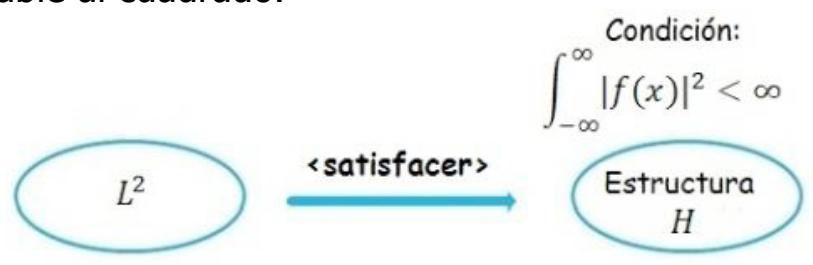

Proposición IV: En 2 está definido un producto escalar. Representado con el siguiente mentefacto proposicional:

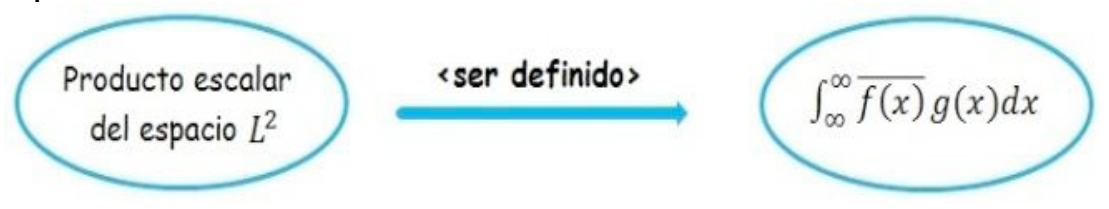




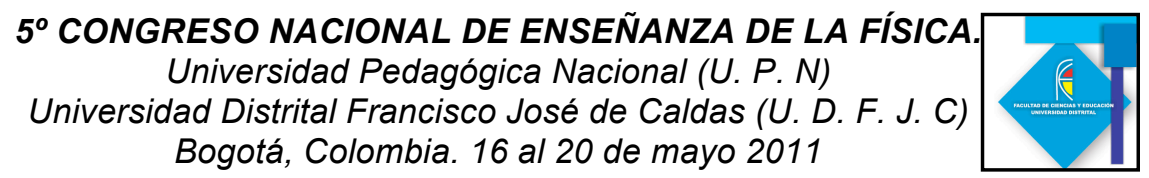

En síntesis, el espacio de Hilbert 2 se constituye en la clase supraordinada de las funciones de onda. A cada estado de un sistema cuántico se le asocia una función de onda.

Supongamos que la función de onda 1 representa un cierto estado, al realizar una medición se llega a un resultado determinado 1 . De igual manera, se supone que 2 representa otro estado, al realizar una medición se llega a un resultado determinado 2. Toda combinación lineal de 111 , es decir, $11+22$ define un estado en el que realizar una medición puede dar el resultado 1 o el resultado 2. Proposición I: La función de onda cumple con el principio de superposición de estados.
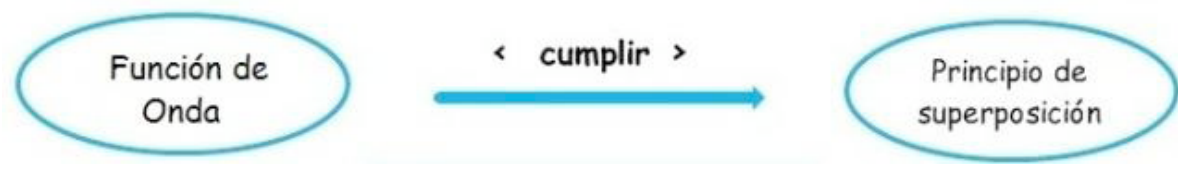

El cuadrado del módulo de esta función 2 se denomina densidad de probabilidad, la cual determina la distribución de probabilidad de los valores permitidos de la magnitud física en cuestión. Proposición II: El cuadrado del valor absoluto de la función de onda es la probabilidad.

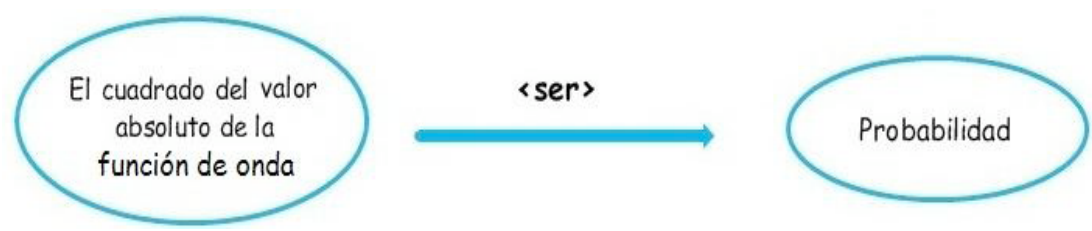

La suma de las probabilidades de todos los valores posibles de las coordenadas de un sistema debe ser, por definición igual a la unidad. De manera que:

$2=1$

Proposición III: La función de onda cumple con la condición de normalización.
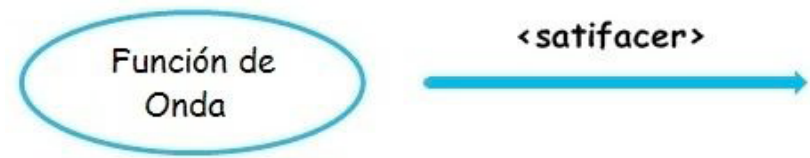

Condición de Normalización

La densidad de probabilidad, satisface la ecuación de continuidad de manera que es posible determinar una corriente de probabilidad, la cual es:

$\equiv-h 2\{\nabla, *-\nabla r, t\}$

Proposición VI: El módulo al cuadrado de la función de onda satisface la ecuación de continuidad.
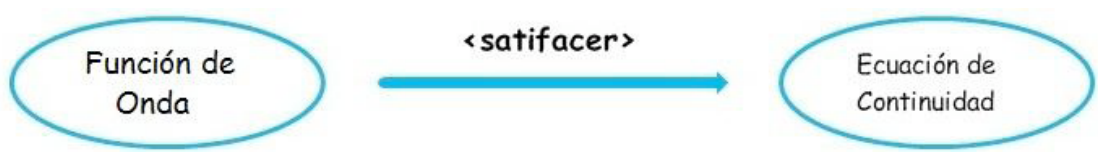

La función de onda es solución de la ecuación de Schrödinger, la cual es una ecuación diferencial de segundo orden y es compleja porque contempla la variable imaginaria .

$$
i \hbar \frac{\partial \varphi(r, t)}{\partial t}=-\frac{\hbar^{2}}{2 m} \frac{\partial^{2} \varphi(r, t)}{\partial x^{2}}+V(r) \varphi(r, t)
$$

Proposición VII: La función de onda satisface la ecuación de Schrödinger. 
$5^{\circ}$ CONGRESO NACIONAL DE ENSEÑANZA DE LA FÍSICA

Universidad Pedagógica Nacional (U. P. N)

Universidad Distrital Francisco José de Caldas (U. D. F. J. C)

Bogotá, Colombia. 16 al 20 de mayo 2011
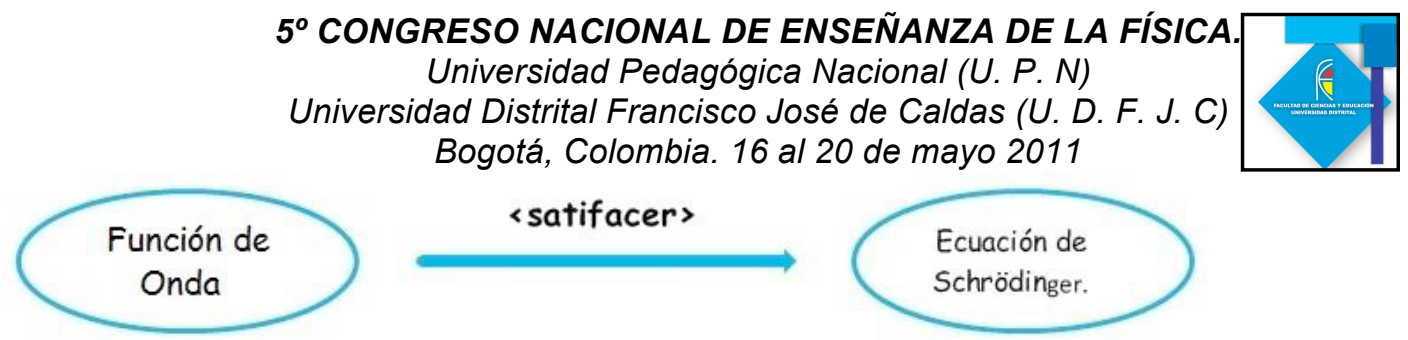

\section{b. Clase Excluida}

Otras funciones que pertenecen 2 y que no tienen las características propias de las funciones de onda se denominan clase excluida. Por ejemplo las soluciones de la ecuación de difusión, por lo tanto las funciones de difusión será nuestra clase excluida. Las funciones de difusión toman valores reales mientras que las funciones de onda tienen necesariamente tienen una parte imaginaria (Eisberg, 1971).

Proposición I: La función de difusión es real.
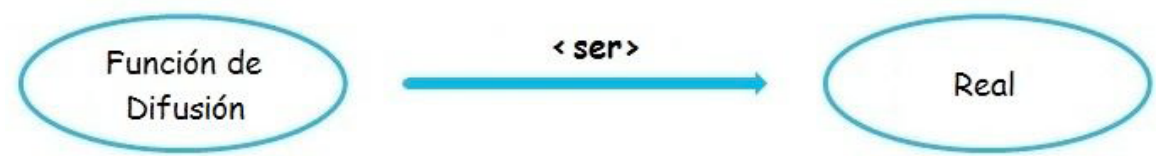

Aunque esas funciones de difusión tienen las mismas propiedades matemáticas de la función de onda, ya que ambas pertenecen 2, difieren en el significado físico. Las funciones de difusión describen eventos como la dispersión gradual de un gas, mientras que la función de onda describe eventos a nivel atómico, como ya se mencionó.

\section{c. Clase Infraordinada: Operadores}

El subconjunto de 2 formado por funciones de onda que están asociadas a un operador, la denominamos la clase infraordinada de la función de onda. A continuación especificaremos un subconjunto 2 de formado por funciones de onda que están asociadas a un operador .

Si la medida de un observable (ejemplo: spin, momento, posición) es discreta, finita de numero $N$, los resultados serán $=1,2, \ldots$ entonces $A$ es una matriz hermítica sobre un espacio de Hilbert de dimensión $N$. Siendo $H$ un espacio lineal $N$ dimensional, y por tanto debe haber un conjunto de vectores independientes $=1,2, \ldots$ que forman un sistema de base ortonormal. Relacionados así: $=$. Si deseamos saber la acción del observable en un sistema tenemos que satisfacer $=()$, siendo $f(x)$ la función de onda asociada a la partícula y al operador. Ahora bien si queremos encontrar el subconjunto de todas las funciones de onda que son dominio del operador $D(Q)$ tendríamos: $=\left\{\begin{array}{lll}-\infty & -\infty & 2 \leq \infty\end{array}\right.$

De tal manera que el conjunto de las funciones de onda asociadas a un operador $Q$ es una sub clase de 2 .

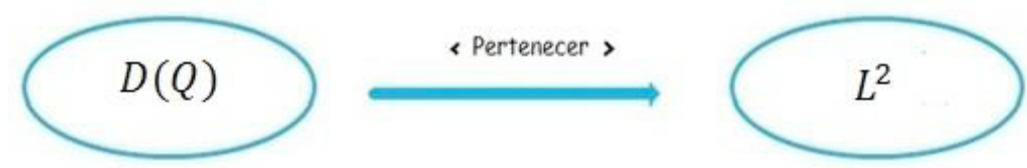

Así mismo podríamos hacer con cada uno de los observables. 
$5^{\circ}$ CONGRESO NACIONAL DE ENSEÑANZA DE LA FÍSICA

Universidad Pedagógica Nacional (U. P. N)

Universidad Distrital Francisco José de Caldas (U. D. F. J. C)

Bogotá, Colombia. 16 al 20 de mayo 2011

\section{MENTEFACTO CONCEPTUAL PARA LA FUNCIÓN DE ONDA}

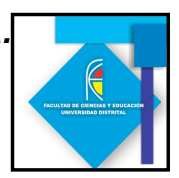

El diagrama que se presenta a continuación es el mentefacto conceptual para la función de onda.

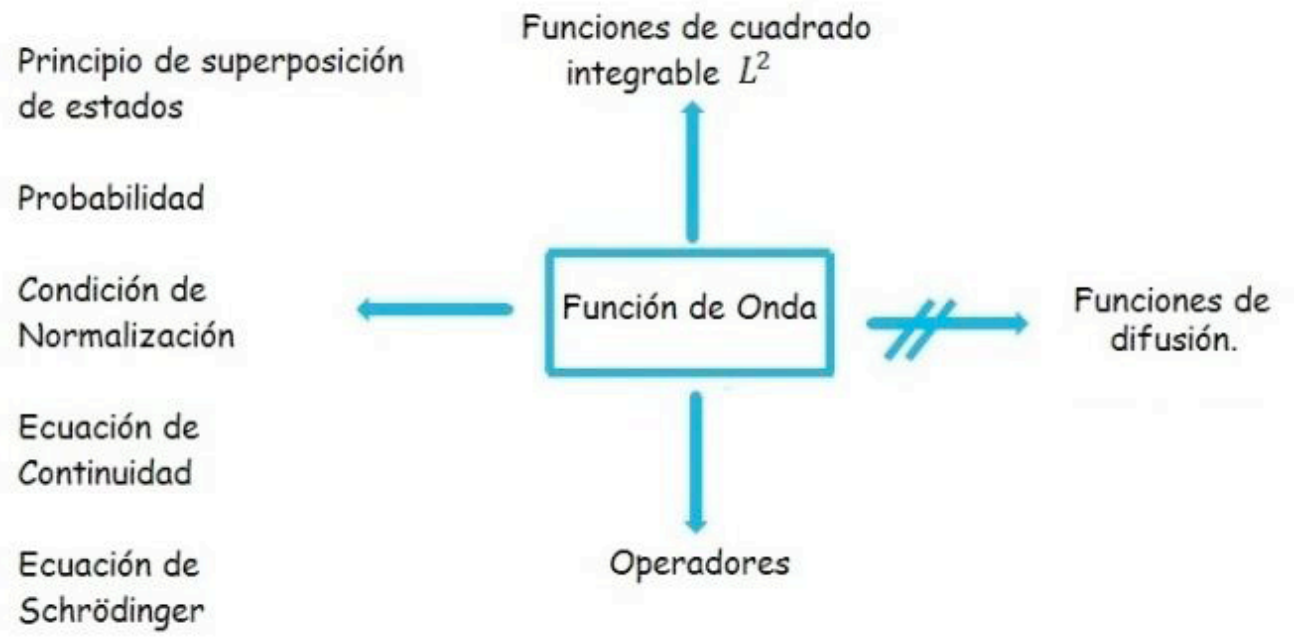

\section{CONCLUSIONES}

- Es posible analizar un concepto por medio de diagramas cognitivos como los mentefactos conceptuales.

- Los mentefactos conceptuales permitieron identificar y organizar las nociones constitutivas y diferenciadoras del concepto de función de onda, tales como: Las funciones de onda pertenecen a , son complejas y describe fenómenos atómicos, y aunque otras funciones como la función de difusión pertenecen a describe otro tipo de fenómenos y son reales.

- Los diagramas cognitivos como los mentefactos conceptuales son herramientas para el aprendizaje de un concepto ya que propicia las operaciones cognitivas propias del pensamiento conceptual.

\section{BIBLIOGRAFIA}

Bao, L. (1999). Using the Context of Physics Problem Solving to Evaluate the Coherence of Student Knowledge. Tesis Doctoral para la obtención del título de Doctor en Filosofía de la Física. Departamento de Física, Universidad de Maryland, Estados Unidos.

De la Madrid, R. (2001). Quantum Mechanics in Rigged Hilbert Space Language. Tesis Doctoral para la obtención del título de Doctor en Filosofía de la Física. Departamento de Física Teórica. Universidad del País Vasco. Bilbao. España.

De la Madrid, R. (2005). The role of the rigged Hilbert space in the quantum Mechanics, Departamento de Física Teórica, ArXiv. 1, 9. 


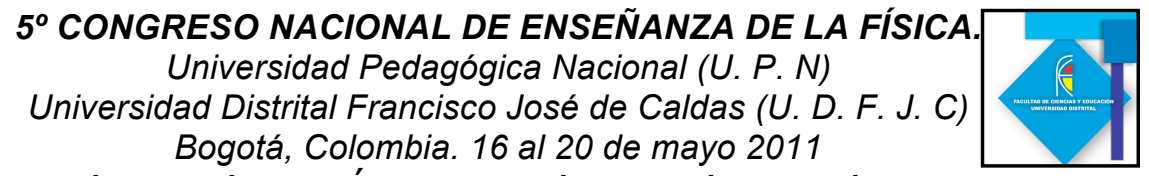

Eisberg, R. (2006). Física Cuántica: Átomos moléculas, sólidos, núcleos partículas. México. Limusa, Noriega Editores.

Greca, I. (2002). Construyendo significados en mecánica cuántica: Fundamentación y resultados de una propuesta innovadora para su introducción en el nivel universitario, Enseñanza de las ciencias. 20, 2, 327-338.

Orlando, O. (2007). Una idea profunda en la comprensión del mundo físico: el principio de superposición de estados, Latin american journal physic education, 1,1 , 83-88.

Ortuño, M. Serrano, M. (2004). Física Cuántica. Universidad de Murcia (Notas de Clase). Murcia: España.

Schrödinger, E. (1935). Discussion of Probability Relations Between Separated Systems. Proceedings of the Cambridge Philosophical Society. 31, 555-662.

Zubiría, M. (1999). Tratado de pedagogía conceptual: Mentefactos I. Bogotá. 